\title{
2(+1?) Binary Central Stars: A Spectroscopic Study
}

\author{
Katrina Exter, Don Pollacco \\ APS Division, Physics, Queen's University Belfast, Belfast, BT7 1NN, \\ $U K$
}

\begin{abstract}
We report on a study of 3 post common-envelope binary systems; EC 11575-1845, V644 Cas (the central star of the PN HFG 1), and VW Pyx (the central star of K 1-2). These 3 have similar photometric and spectroscopic characteristics. Emission lines from the heated face of the cool star move with different velocities, and the $\mathrm{H}$ I lines are very broad with deep absorption component(s) visible at all phases.
\end{abstract}

\section{EC 11575-1845}

The rich set of emission lines trace the motion of the irradiated face of the cool component, but the percentage modulation of their flux exceeds that of the reflection effect (from Chen et al., 1995). The $\mathrm{H}$ I lines are broad and orbit faster than the other emission lines, and show deep absorption that moves in phase but with a slower velocity. Absorption lines of $\mathrm{H} \mathrm{I}$ and He II from the sdO star are visible, and reversed emission (a consequence of high temperature) in He II allows its orbital velocity to be determined. With these information we place limits on the masses and radii of the two components (Exter, Pollacco, Bell, Napiwotzki \& Maxted, in preparation).

\section{V644 Cas}

V644 Cas shows absorption and emission lines from the cool star, and absorption (with reversed emission in He II) from the hot star, allowing for tighter limits on the stellar parameters. The spectra are similar to that of EC 11575-1845, with differential velocities, however the absorption in the $\mathrm{H} \alpha$ profile appears to have two components, in this case moving together with the emission.

\section{VW Pyx}

The spectra are similar to those for the other two systems, although it is more difficult to determine if differential velocities are to be found. We also find absorption components in the $\mathrm{H}$ I profiles. This and more will be reported on in Exter, Pollacco, \& Bell (in preparation).

\section{References}

Chen, A., O'Donoghue, D., Stobie, R. S., Kilkenny, D., Roberts, G., van Wyk, F., 1995, MNRAS, 275, 100 